\title{
Ventilación no invasiva en infección respiratoria aguda fuera del área de cuidado intensivo
}

\author{
BERNARDO ALONSO C. ${ }^{1}$, MARIE BOULAY S. ${ }^{2}$, PATRICIA DALL'ORSO V. ${ }^{3}$, \\ GUSTAVO GIACHETTO L. ${ }^{4}$, AMANDA MENCHACA N. ${ }^{5}$, MARÍA CATALINA PIREZ G. ${ }^{6}$ \\ 1. Asistente de Clínica Pediátrica, Facultad Medicina, Universidad de la República Uruguay. \\ 2. Jefa de Residentes de Pediatría. Universidad de la República Uruguay. \\ 3. Profesora Adjunta Clínica Pediátrica, Facultad Medicina, Universidad de la República Uruguay. \\ 4. Profesor Clínica Pediátrica, Facultad Medicina, Universidad de la República Uruguay. Subdirector Departamento \\ de Pediatría y Especialidades. \\ 5. Profesora Unidad de Cuidado Intensivo de Niños, Facultad Medicina, Universidad de la República Uruguay. \\ 6. Profesora Clínica Pediátrica, Facultad Medicina, Universidad de la República Uruguay.
}

\begin{abstract}
Non-invasive ventilation in patients with acute respiratory infection outside of intensive care units

Introduction: Acute Respiratory Infections (ARIs) in children constitute a Public Health issue. They represent the main cause of admission to hospitals and ICU use throughout the winter months. The benefit of noninvasive ventilation (NIV) in its treatment is not clearly defined. Objective: Describe the results of use of NIV in hospital, outside of ICU in children with severe ARIs. Patients and Methods: Population included all children $<2$ y.o. with probable or confirmed viral ARI with Tal score $>8$ o $>6$ without previous treatment response; all these seen between 8 June and 20 October, 2009. A management protocol was established, including monitoring and connection to NIV. A Vipap III (ResMed(R) was used. Clinical characteristics and evolution of patients is described. Results: 113 patients were included, which amounted to 5.2 of all admissions for ARI in that period. $80.5 \%(\mathrm{n}=91)$ showed a favorable evolution, with an average LOS of $76.1 \mathrm{hrs}$. The rest required conventional mechanical ventilation. No patient died. Conclusions: The use of NIV among these children is a valid and secure option. It can also be utilized outside of the ICU so long as it is limited to a restricted area, a management protocol, and trained staff.
\end{abstract}

(Key words: Respiratory Infection, non-invasive ventilation).

Rev Chil Pediatr 2011; 82 (3): 211-217

Trabajo recibido el 20 de octubre de 2010, devuelto para corregir el 20 de diciembre de 2010, segunda versión el 29 de diciembre de 2010, aceptado para publicación el 06 de enero de 2011.

Correspondencia a:

Dr. Bernardo Alonso L.

E-mail:alozur@gmail.com 


\section{RESUMEN}

Introducción: La infección respiratoria aguda (IRA) en niños constituye un problema de salud pública. Durante los meses de invierno representa la primera causa de ingreso hospitalario y de demanda de cuidado intensivo. El beneficio de la ventilación no invasiva (VNI) en su tratamiento no ha sido claramente definido. Objetivo: Describir los resultados de la aplicación de ventilación no invasiva fuera de la unidad de cuidado intensivo a niños con infección respiratoria aguda grave. Pacientes y Método: Se incluyeron niños $\leq 2$ años con IRA de etiología viral probable o confirmada y score de Tal $\geq 8$ o $\geq 6$ sin respuesta al tratamiento previo entre 8 de junio y 20 de octubre de 2009. Se estableció un protocolo de actuación, monitorización y conexión a VNI. Se utilizo un Vipap III (ResMed $\left.{ }^{\circledR}\right)$. Se describen las características clínicas y evolutivas de los pacientes. Resultados: Se incluyeron 113 pacientes, el 5,2\% de los ingresos hospitalarios por IRA en ese período. El $80,5 \%(n=91)$ evolucionó favorablemente, con una estadía promedio de 76,1 horas. El resto requirió asistencia ventilatoria mecánica convencional. Ningún paciente falleció. Conclusiones: La aplicación de VNI en éste grupo de niños, constituyó una alternativa válida y segura. Su utilización puede ser extendida fuera del área de cuidado intensivo, siempre que se disponga de un área específica, protocolo de actuación y un equipo previamente capacitado.

(Palabras clave: Infección respiratoria, ventilación no invasiva, VNI, ventilación mecánica).

Rev Chil Pediatr 2011; 82 (3): 211-217

\section{Introducción}

La infección respiratoria aguda (IRA) constituye un problema de salud pública. En Uruguay durante los meses de invierno representa la primera causa de ingreso hospitalario aumentando la demanda de cuidado intensivo. Corresponde a una de las primeras causas de mortalidad infantil y la primera causa de muerte hospitalaria ${ }^{1,2}$.

La ventilación no invasiva (VNI), es una modalidad de soporte ventilatorio que evita la intubación orotraqueal, y por lo tanto, sus riesgos y complicaciones Aplicada inicialmente en adultos con patología crónica, actualmente su uso se ha extendido a niños ${ }^{3-12}$. En los últimos años se han comunicado experiencias de uso de VNI en salas de emergencia

En nuestro medio se inicio su aplicación en las unidades de cuidado intensivo (UCI), principalmente en el tratamiento de descompensaciones agudas de pacientes con hipoventilación crónica y en el destete de la ventilación mecánica prolongada ${ }^{10}$.

Existen comunicaciones de pequeñas series de casos que señalan beneficios con la aplicación de la VNI en el tratamiento de niños con IRA y falla respiratoria aguda moderada ${ }^{13-22}$. La mayoría de los niños con IRA grave presen- tan obstrucción espiratoria de la pequeña vía, atrapamiento aéreo e hiperinsuflación dinámica, aumento del trabajo respiratorio e hipoxemia de grado variable. La VNI brinda apoyo a la musculatura respiratoria, favorece el reclutamiento alveolar y contrarresta la hiperinsuflación dinámica, lo que contribuiría a mejorar la ventilación y la oxigenación. Se postula que su aplicación en forma precoz evitaría la ventilación mecánica.

En el año 2009 en el marco del Plan de Invierno se decidió incorporar la VNI a la asistencia de niños hospitalizados con IRA en un área del sector de cuidados moderados con el propósito de dar respuesta al principal problema asistencial en el Hospital Pediátrico del Centro Hospitalario Pereira Rossell (HP- CHPR).

\section{Objetivo}

Describir los resultados de la aplicación de ventilación no invasiva fuera de la unidad de cuidado intensivo niños con infección respiratoria aguda grave.

\section{Pacientes y Método}

El presente es un estudio prospectivo, en el período comprendido entre el 8 de junio y el 20 de octubre de 2009. 
Para la implementación del soporte ventilatorio no invasivo se dispuso de dos salas contiguas, cada una con tres camas en el área de cuidados moderados próximas a la UCI.

Cada unidad fue equipada con oxígeno y aspiración central, oximetría de pulso y un equipo específico de VNI, Vipap III (ResMed) con humidificador y se utilizó interfase buconasal.

La asistencia estuvo a cargo de personal contratado: pediatras y residentes de pediatría durante las 24 horas; licenciadas de enfermería (una por turno); auxiliares de enfermería ( 3 por turno); y licenciadas en fisioterapia. Docentes de las Clínicas Pediátricas de la Facultad de Medicina y especialistas en cuidado intensivo de niños supervisaron la asistencia.

Previo a la implementación de la Unidad, el personal recibió capacitación en base a talleres sobre temas específicos; insuficiencia respiratoria, ventilación no invasiva, y manejo de vía aérea.

La valoración clínica de la severidad de las IRA se realizó mediante score de Tal, utilizado en las pautas nacionales ${ }^{23,24}$.

Los criterios de ingreso a la Unidad fueron: niños $\leq 2$ años cursando IRA de etiología viral probable o confirmada grave. Se consideró criterio de gravedad score de Tal $\geq 8, o \geq 6 \sin$ respuesta al tratamiento previo.

Se definió IRA de probable etiología viral por la presencia de criterios clínicos (polipnea y/o tiraje acompañada o no de sibilancias, estridor y estertores subcrepitantes bilaterales y difusos), y criterios radiológicos (infiltrado intersticial difuso y/o hiperinsuflación).

La identificación etiológica se realizó mediante la detección de antígenos virales para virus respiratorio sincisial (VRS), influenza A, adenovirus, por inmunocromatografía en muestras obtenidas de aspirado nasofaríngeo en el Laboratorio de Microbiología. La tipificación de influenza A H1N1 pandémico se realizó por PCR en tiempo real en el Laboratorio de Higiene Pública del Ministerio de Salud Publica (MSP).

Se consideraron criterios de exclusión: peso $<6 \mathrm{~kg}$; hemodinamia inestable; depresión neuropsíquica; complicaciones mecánicas (neumotórax, neumomediastino); acidosis mixta con $\mathrm{pH}<7,20$, y $\mathrm{pCO}_{2}>60 \mathrm{mmHg}$.

Se estableció un protocolo de actuación con medidas de estabilización, monitorización clínica, paraclínica, y conexión a VNI. A todos se les colocó sonda nasogástrica.

Se fijaron como parámetros de inicio modo ST; EPAP 4; PS 5; frecuencia respiratoria de respaldo de 20, con un tiempo inspiratorio de seguridad entre 0,6 y 1 segundo y tiempo de subida o rampa (rise time) $150 \mathrm{~ms}$. El flujo inicial de oxígeno fue $5 \mathrm{~L} / \mathrm{min}$. Se realizó sedación con lorazepam $0,05 \mathrm{mg} / \mathrm{kg}$ cada $4 \mathrm{~h}$. Se utilizaron parches hidrocoloides para minimizar fugas y evitar lesiones cutáneas.

Todos los niños recibieron en función de la respuesta clínica y según criterio del medico tratante salbutamol inhalado $(0,15 \mathrm{mg} / \mathrm{kg})$ intercalando el nebulizador en la rama inspiratoria. En caso de respuesta positiva al salbutamol (descenso de al menos dos puntos en el score de Tal) este se continuó cada 4 ó 6 horas.

Se clasificó la insuficiencia respiratoria en tipo I con hipoxia, y tipo II hipoxia e hipercapnia.

Se realizó un seguimiento clínico, valorando la tolerancia a la máscara, sincronización paciente-ventilador, score de Tal, y saturación de oxígeno de la hemoglobina. Se vigilaron parámetros del equipo: volumen corriente, frecuencia respiratoria espontánea, apneas, flujos $\mathrm{y}$ alteraciones en la gasometría arterial.

Se consideraron criterios de interrupción de la técnica, la intolerancia de la máscara, empeoramiento radiológico (extensión del compromiso parenquimatoso o intersticial) y/o la presencia de criterios de fracaso terapéutico. Se consideró fracaso terapéutico: no mejoría clínica con desaturación y/o disminución, hipercapnia severa; escala de Glasgow $<8$; presencia de complicaciones mecánicas (neumotórax, neumomediastino); deterioro clínico y/o ausencia de mejoría a pesar de utilizar máximos recursos terapéuticos (PS 12, EPAP $\left.10, \mathrm{FIO}_{2}>0,6\right)$; inestabilidad hemodinámica $\mathrm{o}$ acidemia con $\mathrm{pH}<7,20$.

En caso de estabilidad clínica y gasométrica, la VNI se mantuvo durante al menos $48 \mathrm{hrs}$ considerando el período de estado de la enfermedad viral.

El destete se programó con score de Tal $<6$. 
Se caracterizaron los pacientes de acuerdo a edad, sexo, peso, diagnóstico y evolución (duración de la ventilación, complicaciones, necesidad de ingreso a UCI, ventilación mecánica, muerte). Se compararon los pacientes con éxito versus con fracaso terapéutico.

\section{Análisis estadístico}

Para las variables continuas los resultados se expresaron como media y rango, y para su comparación se utilizó el test no paramétrico de Mann- Whitney. Para las variables discretas se utilizó el test de $\chi^{2}$ de Pearson. El análisis fue realizado mediante el programa Epi. Info. ${ }^{\text {TM }}$ Versión 3.5.1 (2009). Se consideró significativo $\mathrm{p}<0,05$.

\section{Resultados}

En el año 2009 las IRA representaron el $19,6 \%$ del total de ingresos al HP-CHPR ( $\mathrm{n}=$ 426). El 5,2\% $(\mathrm{n}=113)$ cumplieron con los criterios de ingreso y recibieron VNI fuera de la UCI.

En la tabla 1 se resumen las características clínicas al ingreso de los niños que recibieron soporte de VNI.

La mayoría eran varones $65,4 \%$ de 6 a 12 meses $(57,5 \%)$. El diagnóstico más frecuente fue bronquiolitis $(59,2 \%)$.

El 80,5\% $(\mathrm{n}=91)$ de los niños evolucionó en forma favorable, con una estadía promedio en la unidad de 76,1 horas (rango 10-88). El $19 \%(n=22)$ requirió traslado a unidad de cuidado intensivo para asistencia ventilatoria mecánica invasiva (AVMI). En estos casos el promedio de estadía en la unidad de VNI fue 21 horas (rango 4-40). Los criterios para la suspensión de VNI fueron: intenso trabajo respiratorio mantenido a pesar de utilizar los máximos parámetros de soporte ventilatorio preestablecidos $(\mathrm{n}=11)$, acidosis respiratoria $(\mathrm{n}=9)$, acidosis metabólica grave $(\mathrm{n}=1)$. Un paciente presento como complicación neumotórax no hipertensivo.

Al comparar los niños con evolución favorable con aquellos que requirieron AVMI no se observaron diferencias en relación a edad, sexo, peso, diagnóstico al ingreso y agente etiológico (tabla 2).
Tabla 1. Características clínicas al ingreso de los niños que recibieron VNI. Hospital Pediátrico Centro Hospitalario Pereira Rossell 8/6 - 20/10/2009 ( $n=113)$

\begin{tabular}{|lrc|}
\hline Característica & n & $\mathbf{( \% )}$ \\
Edad & & \\
$<6$ meses & 25 & $(22,1)$ \\
6 a 12 meses & 65 & $(57,5)$ \\
13 a 24 meses & 23 & $(20,3)$ \\
Sexo & & \\
Masculino & 74 & $(65,4)$ \\
Diagnóstico & & \\
Bronquiolitis & 67 & $(59,2)$ \\
SBOL* & 14 & $(12,3)$ \\
Neumonitis & 21 & $(18,5)$ \\
Neumonía & 11 & $(9,7)$ \\
Antígeno viral & & \\
VRS & 60 & $(53,1)$ \\
Influenza A & 17 & $(15)$ \\
VRS/Influenza A & 4 & $(3,5)$ \\
Influenza A + Adenovirus & 2 & $(1,7)$ \\
VRS / Adenovirus / Influenza A & 1 & $(0,8)$ \\
Negativo & 29 & $(25,6)$ \\
\hline
\end{tabular}

* Síndrome broncobstructivo del lactante: dos o más crisis broncobstructivas.

Tabla 2. Características de los niños y evolución clínica. Hospital Pediátrico Centro Hospitalario Pereira Rossell 8/6 - 20/10/2009 $(n=113)$

\begin{tabular}{|c|c|c|c|}
\hline & $\begin{array}{c}\text { Evolución } \\
\text { favorable } \\
(\mathbf{n}=91)\end{array}$ & $\begin{array}{c}\text { Requirió } \\
\text { AVMI } \\
(n=22)\end{array}$ & $\mathbf{p}$ \\
\hline $\begin{array}{l}\text { Edad (meses) } \\
\text { Media } \\
\text { Mediana } \\
\text { Rango }\end{array}$ & $\begin{array}{c}9,43 \\
9 \\
2 \text { a } 21\end{array}$ & $\begin{array}{c}9,23 \\
9 \\
3 \text { a } 16\end{array}$ & NS \\
\hline $\begin{array}{l}\text { Sexo masculino } \\
\mathrm{n}(\%)\end{array}$ & $56(61,5)$ & $18(81,8)$ & NS \\
\hline $\begin{array}{l}\text { Peso }(\mathrm{kg}) \\
\text { Media } \\
\text { Mediana } \\
\text { Rango }\end{array}$ & $\begin{array}{c}8,51 \\
8,30 \\
6,3-12\end{array}$ & $\begin{array}{c}8,90 \\
9,00 \\
6,3-2,10\end{array}$ & NS \\
\hline \begin{tabular}{ll}
\multicolumn{2}{c}{ Diagnóstico } \\
BQ & $n(\%)$ \\
SBOL & $n(\%)$ \\
NA & $n(\%)$ \\
NI & $n(\%)$
\end{tabular} & $\begin{array}{r}52(57,1) \\
13(14,3) \\
8(8,8) \\
18(19,8)\end{array}$ & $\begin{array}{r}15(68,1) \\
1(4,5) \\
3(13,6) \\
3(13,6)\end{array}$ & $\begin{array}{l}\text { NS } \\
\text { NS } \\
\text { NS } \\
\text { NS }\end{array}$ \\
\hline $\begin{array}{l}\text { Antigenos virales } \\
\text { VRS } \\
\text { Influenza A } \\
\text { IA / Adenovirus } \\
\text { IA / VRS } \\
\text { IA / VRS / AD } \\
\text { Negativo }\end{array}$ & $\begin{array}{r}47(51,6) \\
15(16,5) \\
2 \quad(2,2) \\
2 \quad(2,2) \\
1 \quad(1,1) \\
24(26,4)\end{array}$ & $\begin{array}{c}13(59,1) \\
2 \quad(9,1) \\
== \\
2(9,1) \\
=== \\
5(22,7)\end{array}$ & $\begin{array}{l}\text { NS } \\
\text { NS } \\
\text { NS } \\
\text { NS } \\
\text { NS } \\
\text { NS }\end{array}$ \\
\hline
\end{tabular}

BQ: Bronquiolitis, SBOL: Síndrome broncobstructivo del lactante 2 o más crisis broncobstructivas NA: Neumonía con consolidación, NI: Neumonitis intersticial IA: Influenza A AVMI: ventilación invasora. 
Tabla 3. Severidad al ingreso de los niños tratados con VNI con evolución favorable vs los niños que requirieron AVMI .Hospital Pediátrico Centro hospitalario Pereira Rossell 8/6 - 20/10/2009 $(n=113)$

\begin{tabular}{|c|c|c|c|}
\hline & $\begin{array}{l}\text { Evolución favorable } \\
(n=91)\end{array}$ & $\begin{array}{l}\text { Requirieron AVMI } \\
(n=22)\end{array}$ & $\mathbf{P}$ \\
\hline $\begin{array}{l}\text { Escore de Tal n (\%) } \\
\leq 9 \\
\geq 10 \\
\text { s/d }\end{array}$ & $\begin{array}{ll}84 & (92 \%) \\
7 & (7,6 \%)\end{array}$ & $\begin{aligned} 18 & (85,7 \%) \\
3 & (14,2 \%) \\
1 & \end{aligned}$ & NS \\
\hline $\begin{array}{l}\text { Frecuencia respiratoria (rpm) n (\%) } \\
\leq 80 \\
>80 \\
\text { s/d }\end{array}$ & $\begin{array}{l}70(76,9 \%) \\
15(16,4 \%) \\
6\end{array}$ & $\begin{array}{rr}18 & (81,8 \%) \\
2 & (9,0 \%) \\
2 & \end{array}$ & NS \\
\hline $\begin{array}{l}\text { Frecuencia cardíaca (cpm) n (\%) } \\
\leq 170 \\
>170 \\
\text { S/d }\end{array}$ & $\begin{aligned} 71 & (78 \%) \\
5 & (5,4 \%) \\
15 & \end{aligned}$ & $\begin{aligned} & 13(59 \%) \\
& 6(27,2 \%) \\
& 3\end{aligned}$ & $<0,01$ \\
\hline $\begin{array}{l}\text { Tipo de insuficiencia respiratoria } \\
\text { Tipo I (hipoxémica) } \\
\text { Tipo II (hipercapnica e hipoxémica) }\end{array}$ & $\begin{array}{l}75(84 \%) \\
16(17 \%)\end{array}$ & $\begin{aligned} 15 & (68,2 \%) \\
7 & (31,8 \%)\end{aligned}$ & $\begin{array}{l}\text { NS } \\
\text { NS }\end{array}$ \\
\hline
\end{tabular}

VNI: ventilación no invasiva, AVMI: ventilación invasiva.

Al comparar entre estos grupos, la severidad de la enfermedad al ingreso evaluada mediante score de Tal, frecuencia respiratoria, y tipo de insuficiencia respiratoria, no se encontraron diferencias significativas. En el grupo que requirió asistencia ventilatoria invasiva se observo una proporción mayor de niños con frecuencia cardíaca mayor a $170 \mathrm{cpm}(\mathrm{p}<$ 0,05) (Tabla 3).

En esta serie no se registraron muertes.

\section{Discusión}

A pesar de los avances en el conocimiento sobre los agentes responsables de las IRA virales, el tratamiento actual de las formas graves continúa basándose en la aplicación de oxígeno y soporte ventilatorio.

En los últimos años se han reportado resultados alentadores con la aplicación de VNI a niños con insuficiencia respiratoria en área de cuidado intensivo ${ }^{25-31}$.

Esta representa la primera experiencia en Uruguay de la aplicación de VNI en el tratamiento de niños con IRA grave fuera de la UCI.

Este estudio presenta limitaciones derivadas del escaso número pacientes incluidos y del diseño metodológico ya que se trata de un estudio descriptivo de casos. La validación de una alternativa terapéutica exige la utilización de un grupo control y la planificación de un ensayo terapéutico.

Independientemente de estas limitaciones, se puede afirmar que en esta serie la aplicación de VNI resultó beneficiosa en la mayoría de los niños con IRA grave.

Es posible que el beneficio terapéutico observado este vinculado con la utilización de un procedimiento standardizado con criterios de selección claramente definidos, vigilancia permanente de parámetros ventilatorios preestablecidos y capacitación previa de los recursos humanos. Esto ha sido referido por otos autores como la clave del éxito de la VNI en niños con IRA grave ${ }^{27}$. Otros factores que pueden haber contribuido a la alta tasa de evoluciones favorables fue el tipo de paciente en el que la técnica fue aplicada. Las limitaciones técnicas vinculadas al tipo de equipo de ventilación disponible determino que la mayoría de los niños incluidos tuvieran peso mayor a 7 $\mathrm{Kg}$. Algunos autores señalan fracasos de esta técnica con edad y peso menor al utilizado en esta serie ${ }^{26,27}$.

Esta experiencia fue desarrollada coincidiendo con la epidemia de infección por el virus influenza A H1N1 pandémico. El 11 de junio se declaro el pasaje a la fase 6 de pan- 
demia y por tanto, al comprobarse circulación viral sostenida, el Departamento de Vigilancia en Salud del Ministerio de Salud Publica cambió la estrategia de vigilancia epidemiológica pasando de la identificación de casos individuales al monitoreo de la situación epidemiológica, la identificación y control de brotes y la identificación de casos graves ${ }^{32}$. Esto determinó que, en esta serie la confirmación diagnóstica mediante PCR en tiempo real no se realizara a todos los niños hospitalizados en la Unidad con antígenos de influenza $\mathrm{A}$ en muestras de aspirado nasofaríngeo. El diagnóstico de infección por influenza AH1N1 pandémico se confirmó en 3 casos. A pesar de ello, todos los niños con antígenos de influenza $\mathrm{A}$ recibieron tratamiento con oseltamivir oral, ofreciéndoseles además la VNI como modalidad terapéutica alternativa para el fallo respiratorio agudo, respetando estrictamente las medidas de aislamiento por cohorte. A pesar de esta situación epidemiológica la mayoría de los niños que recibieron VNI presentaban bronquiolitis a VRS.

En esta serie, no se observó relación entre diagnóstico al ingreso y tipo de virus identificado con el resultado terapéutico. La búsqueda de una posible relación entre el diagnóstico clínico radiológico y etiológico y el resultado terapéutico exige la inclusión de un número mayor de casos.

Constituye un desafío intentar establecer que pacientes se podrían favorecer y por tanto, que lugar ocuparía ésta modalidad de soporte ventilatorio en el tratamiento de niños con IRA grave.

En esta serie el score de Tal, la frecuencia respiratoria, o el tipo de insuficiencia respiratoria, no se relacionaron con la evolución y necesidad de asistencia ventilaroria mecánica invasiva. El único parámetro que se asoció con necesidad de AVMI fue la taquicardia mayor a $170 \mathrm{cpm}$. El valor predictivo de la taquicardia ha sido puesto en duda en otros estudios ${ }^{27}$. Algunos autores han señalado que la $\mathrm{PaCO}_{2}$ elevada es un factor predictivo de fracaso ${ }^{21}$. Definir factores predictivos de éxito y o fracaso terapéutico de la VNI exige un diseño metodológico diferente con mayor número de pacientes, homogeneidad en la población objeto de estudio, y análisis de otras variables.
Al igual que lo observado en otras series, las complicaciones vinculadas con la VNI fueron poco frecuentes; sólo un niño presentó neumotórax no hipertensivo.

El desarrollo de esta Unidad contribuyó a la formación de recursos humanos en el soporte de la vía aérea y en el diagnóstico y tratamiento del fallo respiratorio agudo. Favoreció además, la integración de pediatras e intensivistas.

Los resultados de esta experiencia motivaron la inclusión de ésta modalidad de tratamiento en la planificación de la futura estrategia "plan de Invierno 2010", promoviendo su aplicación en forma más precoz y oportuna.

Es posible que este tipo de estrategias terapéuticas contribuya a optimizar el tratamiento de los niños con IRA graves, evitando traslados a unidad de cuidados intensivos y la aplicación de AVMI con los riesgos y complicaciones que ello implica.

\section{Referencias}

1.- Ferrari AM, Ferreira A, De Leonardis D, Fernández A, Imbriaco J: Mortalidad hospitalaria en un hospital pediátrico de referencia nacional:Centro Hospitalario Pereira Rossell Rev Med Uruguay 2002; 18: 59-65.

2.- Santoro A, Hackenbruck C, Gutiérrez C, et al: Evolución de la mortalidad en el Hospital Pediátrico del Centro Hospitalario Pereira Rossell en el período 19992002. Informe del Comité de Auditoría de Fallecidos. Arch Pediatr Urug 2003; 74 (3): 212-8.

3.- De la Torre L, Gutiérrez N, Garrido E: Manejo de la bronquiolitis grave con CPAP nasal en la planta de un hospital secundario previo al traslado a UCIP. An Pediatr (Barc) 2008; 68 (2): 1-378. 95.

4.- Bueno F, Piva J, García P, Lago P, Einloft P: Outcome and characteristics of infants with acute viral bronchiolitis submitted to mechanical ventilation in a Brazilian pediatric intensive care. Rev Bras Ter Intensiva 2009; 21 (2): 174-82.

5.- Javouhey E, Barats A, Richard N, Stamm D, Floret D: Non-invasive ventilation as primary ventilatory support for infants with severe bronchiolitis. Intensive Care Med 2008; 34 (9): 1608-14.

6.- Caritg J, Pons M, Palomeque A: Bronquiolitis En: Manual de Cuidados Intensivos Pediatricos. Lopez-Herce J, Calvo C, Lorente M, Jaimovich D. 1ed Ed Publimed. Madrid 2001; 98-104. 
7.- García MÁ, Jiménez R, Alonso A, Abad E: Ventilación no invasiva. En Manual de Cuidados Intensivos Pediátricos.Lopez-Herce J, Calvo C, Baltodano A, Rey C, Rodríguez A, Lorente M. 3ed Ed Publimed. Madrid. 2009; 810-7.

8.- Joshi G, Tobias JD: A five-year experience with the use of BiPAP in a pediatric intensive care unit population. $\mathrm{J}$ Intensive Care Med 2007; 22: 38-43.

9.- Pons M, Gili T: Ventilación no invasiva en pediatría. En Manual de ventilación mecánica en pediatría. 2ed Ed Publimed. Madrid, 2009.

10.- Menchaca A, Mercado S, Alberti M: Aplicación de ventilación no invasiva en el niño. Arch Pediatr Urug 2005; 76 (3): 243-51.

11.- Silva D, Krepel F, Troster EJ: Ventilação não invasiva em pediatria. Pediatr (Rio J) 2003; 79 (Supl. 2): 161-8.

12.- Norregaard O: Noninvasive ventilation in children. Eur Respir J 2002; 20: 1332-42.

13.- Campion A, Huvenne H, Leteurtre S, et al: Ventilation non invasive des nourrissons ayant une infection respiratoire sèvere présumée á virus respiratoire syncitial:faisabilité et critères d'èchec. Arch Pediatr 2006; 13: 1404-9.

14.- Leclerc F, Scalfaro P, Noizet O, Thumerelle C, Dorkenoo $A$, Fourier $C$ : Mechanical ventilatory support in infants with respiratory syncytial virus infection. Pediatric Critical Care Medicine 2001; (2): 197-204.

15.- Soong WJ, Hwang B, Tang R: Continuous positive airway pressure by nasal prongs in bronchiolitis. Pediatr Pulmonol 1993; 16: 163-6.

16.- Medina A, Prieto S, Los Arcos A, et al: Aplicación de ventilación no invasiva en una unidad de cuidados intensivos pediátricos An Pediatr (Barc) 2005; 62(1).

17.- Prado F, Godoy MA, Godoy M, Boza ML: Ventilación no invasiva como tratamiento de la insuficiencia respiratoria aguda en Pediatría. Rev Med Chile 2005; 133: 525-33.

18.- Teague WG: Non-invasive positive pressure ventilation: current status in paediatric patients. Paediatr Respir Rev 2005; 6 (1): 52-60.

19.- Larrar S, Essouri S, Durand P, et al: Effects of nasal CPAP ventilation in infants with severe acute bronchiolitis. Arch Pediatr 2006; 13: 1397-403.

20.- Beasley JM, Jones SEF: Continuous positive airway pressure in bronchiolitis. BMJ 1981; 283: 1506-9.
21.- Sepúlveda J, Carrasco JA, Castillo A, et al: Ventilación no invasiva en Insuficiencia respiratoria aguda. Rev Chil Pediatr 2008; 79 (6): 593-9.

22.- Yáñez L, Yunge M, Emilfork $M$, et al: A prospective, randomized, controlled trial of noninvasive ventilation in pediatric acute respiratory failure. Pediatric Critical Care Medicine 2008; 9 (5): 484-9.

23.- Bronquiolitis. En: Atención Pediátrica. Pautas de diagnóstico, tratamiento y prevención. 6 ed. Montevideo: Fefmur 2007; 143-8.

24.- Síndrome Broncobstructivo del lactante. En: Pediatría urgencias y emergencias Bello Sehabiague Prego de Leonardis 2 ed. Montevideo, ed. Bibliomedica, 2005; 499-507.

25.- Cambonie G, Milési C, Jaber S: Nasal continuous positive airway pressure decreases respiratory muscles overload in young infants with severe acute viral bronchiolitis. Intensive Care Med 2008; 34: 1865-72.

26.- Ghiggi M, Chede C, Saporiti A: CPAP nasofaringeo en el fallo respiratorio agudo pediátrico, un método de ventilación no invasiva adaptado a nuestra economía Med Infantil 2000; 7(4): 26727.

27.- Mayordomo J, Medina A, Rey C, Los Arcos M, Concha, Menéndez A: Predictores de éxito y de fracaso en la ventilación no invasiva en la bronquiolitis aguda. An Pediatr (Barc) 2009; 70 (1): 34-9.

28.- Thia L, McKenzie S, Blyth T: Randomised controlled trial of nasal continuous positive airways pressure (CPAP) in bronchiolitis. Arch Dis Child 2008; 93: 45-7.

29.- Essouri S, Chevert L, Durand P, et al: Noninvasive positive pressure ventilation: five years experience in a PICU. Pediatr Crit Care Med 2006; 7: 329-34.

30.- Padman $R$ : Noninasive nasal mask positive pressure ventilation in a pediatric patient with acute hypoxic respiratory failure Pediatr emerg care 1996;12 44-47 Crit care med 1998; 26: 169-173.

31.- Bernet V, Hug MI, Frey B: Predictive factors for the success of noninvasive mask ventilation in infants and children with acute respiratory failure. Pediatr Crit Care Med 2005; 6 (6): 660-4.

32.- División Epidemiología. Departamento de Vigilancia en Salud. Ministerio de Salud Pública: Informe de la situación de Influenza A H1N1 en Uruguay. 2009. Disponible en: www.presidencia.gub.uy/_.../2009/.../ 2009071702.htm 